\section{The role of acupuncture in the treatment of orthodontic patients with a gagging reflex: a pilot study}

\author{
E. Sari ${ }^{1}$ and T. Sari ${ }^{2}$
}

IN BRIEF

- Acupressure and acupuncture are accepted as reliable methods while taking upper alginate impressions in orthodontic patients with a gagging reflex.

- The use of laser stimulation in the study is an alternative method for children with needle phobia.

- The combination of laser stimulation of CV 24 and acupressure of PC 6 was found more effective than the laser stimulation of CV 24 alone.

\begin{abstract}
The aim of this study was to investigate two different acupuncture approaches in the treatment of orthodontic patients with a gagging reflex (GR). Forty-five patients (mean age $10.1 \pm 2.6$ years) had an upper dental alginate impression taken prior to receiving laser stimulation. GR assessment was evaluated by using the Gagging Severity Index (GSI). Fifteen patients in Group A underwent a red-light soft magnetic field laser stimulation of conception vessel 24 (CV 24) for 1 min. Fifteen other patients in Group B undertook a combination of laser stimulation of CV 24 and acupressure pericardium 6 (PC 6). Group C, which consisted of the remaining 15 patients, formed the placebo group. After the laser stimulation and acupressure, a second impression was taken. The Gagging Prevention Index (GPI) was used to evaluate gagging reflex after the laser stimulation and acupressure. Both GSI and GPI were recorded at three stages of the dental impression taking procedure, ie with an empty impression tray, with a loaded tray and the ability to keep the impression in the mouth until set. A significant decrease in GPI values compared to GSI values, was observed at the three stages of the impression taking process and after the laser stimulation in Group $A$ and $B(p<0.05)$. The average improvement between the GSI and GPI scores was 58.9\% before and after the laser stimulation in Group B 37.9\% in Group A and 11.2\% in Group C. Both acupuncture points CV 24 and PC 6 were found to be effective in controlling GR in orthodontic patients.
\end{abstract}

\section{INTRODUCTION}

The gag reflex is a physiological reaction that occurs in order to prevent foreign objects or noxious material from entering the pharynx, larynx or trachea. ${ }^{1}$ It is controlled by nerve endings situated on the soft palate, the pharynx and the pharyngeal part of the tongue. ${ }^{2}$ The origin of gagging has been categorised as either somatic or psychogenic. In somatic gagging, touching a trigger area induces the reflex. Psychogenic gagging can be induced without direct contact and the sight, sound, smell or even thought of dental treatment can be sufficient to induce the gag reflex in some individuals. ${ }^{1}$

\footnotetext{
${ }^{1 *}$ Orthodontist, Departments of Orthodontics, Kasimpasa Military Hospital, 34420 Istanbul, Turkey; ${ }^{2}$ Physician, Kasimpasa Military Hospital, 34420 Istanbul, Turkey ${ }^{*}$ Correspondence to Dr Emel Sari

Göksu Evleri Begonya caddesi B41-A, 81600

Anadoluhisarı-Beykoz, Istanbul, Turkey

Email:emel_sari@yahoo.com
}

Online article number E19

Refereed Paper - accepted 10 July 2010

DOI: $10.1038 /$ sj.bdj.483

${ }^{\circledR}$ British Dental Journal 2010; 208: E19
In orthodontics, the fabrication of oral appliances requires impressions taken from patients. The reflex is exaggerated in some individuals which makes maxillary impressions extremely difficult. Various methods have been proposed to manage the gag reflex. They include relaxation, distraction and desensitisation techniques; psychological and behavioral therapies; local anesthesia, conscious sedation and general anesthesia techniques and complementary medicine therapies including hypnosis, acupuncture and acupressure.,4

Acupuncture is defined as the insertion of a solid needle into specific parts of the human body for disease prevention, therapy or the maintenance of health. Acupuncture points on the body are stimulated by needles, pressure (acupressure),vacuum (cupping), laser or electrical stimulus (electro-acupuncture). ${ }^{5}$

Acupuncture points have been used as an alternative method to control the gag reflex. Several acupuncture points including pericardium 6 (PC 6), ${ }^{6}$ located on the forearm and conception vessel 24 (CV 24), located in the labio-mental fold on the chin may control the gag reflex. The use of needle acupuncture in children is a very difficult procedure since it is an invasive technique. However laser acupuncture and acupressure are painless and more suitable for children.

Our aim was to compare and contrast the effects of two approaches in orthodontic patients with a gagging reflex (GR). The first approach involved the laser stimulation (CEPES-Laser ${ }^{\varpi}$ AMS GmbH, Tannenweng, Germany mean value $650 \mathrm{~nm}, 9 \mathrm{~Hz}, 0.5 \mathrm{~mW}$ ) of the CV 24 acupuncture point. The second consisted of a combination of the laser stimulation of CV 24 acupuncture point and acupressure on PC 6 using bands.

\section{MATERIALS AND METHODS}

A total of 45 (25 female, 20 male) orthodontic patients participated in this study. The mean age was $10.1 \pm 2.6$ years . The patients were divided into three groups: Group A, B and C. Group A consisted of 15 patients who underwent red-light soft magnetic field laser stimulation of CV 24 for 1 min (Fig 1). The 15 patients in Group 
$\mathrm{B}$ had the combination of laser stimulation of CV 24 and acupressure to PC 6 (Fig 2). Group C, consisted of 15 patients and formed the placebo group. ${ }^{8}$ The location of the placebo point was laterally by radius 6 cun, $(1$ cun is equivalent to the width of the patient's thumb across the interphalangeal joint) above the horizontal fold at the root of the hand (Fig 3). The laser stimulation was also applied on the placebo point. The stimulation of the placebo point was different from the stimulation in the two other groups. According to traditional Chinese medicine, the selected placebo point is used for treating circulatory disorders in the upper extremity. ${ }^{8}$

The patient inclusion criteria included the following:

1. Inability to accept dental treatment on a previous occasion due to a severe gag reflex

2. Current dental treatment which required an upper alginate impression to be taken

3. The patient and parents of the patient able to give informed consent.

The upper dental alginate (Quickalgin, Zeus S.r.l., Italy) impressions were taken before acupuncture and second upper alginate dental impression were obtained after acupuncture had occured. The patients were advised to have a light breakfast before coming to the clinic and the impressions were taken between 9.00 and 10.00am. Before taking the impressions, alginate was prepared for $1 \mathrm{~min} 50 \mathrm{~s}$ and the tray was inserted into the mouth where it remained for $45 \mathrm{~s}$. The tray was removed after the alginate set. When the loaded tray was inserted into the mouth, a red-light soft magnetic field laser stimulation was applied to acupuncture point CV 24 for 1 min in Group A. The skin was cleaned with alcohol before application of the laser stimulation. The laser was applied at a distance of $1 \mathrm{~cm}$ from the laser probe to the skin.

In Group B, the patients worn a band (Sea-Band International, Newport, RI, UK) including a button on each wrist for 20 mins before the impression was taken. The button on the band was placed over the PC 6 (Fig 2). The laser stimulation of the CV 24 acupuncture point was also performed for $1 \mathrm{~min}$ while taking impressions in this group.

Table 1 The results of GSI and GPI scores in Group A. SD :standard deviations, $\mathbf{n}=15$. Significance level $\alpha=0.05\left({ }^{*} p<.0 .05\right)$.

\begin{tabular}{l|l|l|l|l|l}
\multirow{2}{*}{ Stages } & \multicolumn{2}{|l|}{ GSI } & GP & \multirow{2}{*}{} \\
\cline { 2 - 6 } & Mean & SD & Mean & SD & \\
\hline Stage I & 2.7 & 0.65 & 1.5 & 0.45 & $0.002^{*}$ \\
\hline Stage II & 3.57 & 0.55 & 2.75 & 1.75 & $0.001^{*}$ \\
\hline Stage III & 4.2 & 0.5 & 2.7 & 1.4 & $0.002^{*}$ \\
\hline $\begin{array}{l}\text { Stage I: when the empty tray was tried in the mouth } \\
\text { Stage II: when the loaded tray was inserted into the mouth } \\
\text { Stage III: when the impression was completed }\end{array}$ & & & \\
\hline
\end{tabular}

Table 2 The results of GSI and GPI scores in Group B. SD: standard deviations, $\mathbf{n}=15$. Significance level $\alpha=0.05\left({ }^{*} \mathrm{p}<0.05\right)$

\begin{tabular}{l|l|l|l|l|l}
\multirow{2}{*}{ Stages } & GSI & GP & \multirow{2}{*}{} \\
\cline { 2 - 5 } & Mean & SD & Mean & SD & \\
\hline Stage I & 3.4 & 0.63 & 1.2 & 0.45 & $0.001^{*}$ \\
\hline Stage II & 4.3 & 0.57 & 1.75 & 0.85 & $0.001^{*}$ \\
\hline Stage III & 4.6 & 0.45 & 1.1 & 0.55 & $0.001^{*}$ \\
\hline $\begin{array}{l}\text { Stage I: when the empty tray was tried in the mouth } \\
\text { Stage II: when the loaded tray was inserted into the mouth } \\
\text { Stage II: when the impression was completed }\end{array}$ & & & \\
\hline
\end{tabular}

Table 3 The results of GSI and GPI scores in Group C. SD: standard deviations, $\mathbf{n}=15$. Significance level $\alpha=0.05\left({ }^{*} \mathrm{p}<0.05\right)$

\begin{tabular}{l|l|l|l|l|l}
\hline \multirow{2}{*}{ Stages } & GSI & GPI & P \\
\cline { 2 - 6 } & Mean & SD & Mean & SD & \\
\hline Stage I & 3.6 & 0.56 & 2.6 & 0.65 & $0.006^{*}$ \\
\hline Stage II & 3.9 & 0.66 & 3.5 & 1.1 & 0.16 \\
\hline Stage III & 4.3 & 0.45 & 4.1 & 0.95 & 0.083 \\
\hline $\begin{array}{l}\text { Stage I: when the empty tray was tried in the mouth } \\
\text { Stage II: when the laded tray was inserted into the mouth } \\
\text { Stage III: when the impression was completed }\end{array}$
\end{tabular}

Table 4 The percentage improvement (\%) between the GSI and GPI scores before and after the acupuncture in the Groups

\begin{tabular}{|l|l|l|l} 
& Group A & Group B & Group C \\
\hline Stage I & 34.4 & 58.9 & 21.7 \\
\hline Stage II & 37.4 & 55.1 & 7.7 \\
\hline Stage III & 41.7 & 62.7 & 4.3 \\
\hline Average improvement & 37.9 & 58.9 & 11.2
\end{tabular}

\section{The concept of laser acupuncture}

Laser acupuncture, which was first scientifically tested in Graz, Austria, and represents a new painless and non-invasive acupuncture method whereby individual combinations of acupuncture points can be stimulated simultaneously as in traditional Chinese medicine. ${ }^{8}$ A search of the literature revealed that laser acupuncture with different wavelengths has been used in several areas, including the treatment of headache ${ }^{9}$ and chronic maxillary sinusitis in children, ${ }^{10}$ anesthesia for minor operations, ${ }^{11}$ therapy relating to acute obstruction in asthmatic children ${ }^{12}$ and vomiting. ${ }^{13}$

In this study, a red-light soft laser (power output $0.5 \mathrm{~mW}$, wavelength $650 \mathrm{~nm}$ ) was used which had a pulsating magnetic field (9 Hz) with a penetration depth of $30 \mathrm{~cm}$ (important in the treatment of scarring with a very high penetration depth into the organism; the pulsating magnetic field permanently supports the laser effect). A redlight soft laser stimulates the organism's 
bioenergetic regulation processes on the cellular level. Litscher ${ }^{8}$ investigated the peripheral effects of acupuncture using a continuous laser light at a wavelength of 685 and $785 \mathrm{~nm}$. A significant increase was observed in peripheral temperature during laser needle acupuncture. In addition flux (product of mean blood flow velocity and concentration of red blood cell) increased markedly 2 min after activation, reaching a maximum at the end of laser needle stimulation. The mean arterial blood pressure did not change significantly and the oxyhaemoglobin level showed an increase. ${ }^{14}$

\section{Evaluation of the gagging reflex}

The Gagging Severity Index (GSI) ${ }^{15}$ was used to assess the magnitude of gag reflex in both treatment groups before the application of acupuncture. The upper dental impression was taken and the Gagging Prevention Index (GPI) ${ }^{15}$ was used to evaluate the effectiveness of the laser acupuncture. Both indexes were based on a descriptive scale of the severity of gag reflex and the ability to carry out upper dental impression.GSI and GPI were recorded at three stages of the impression taking procedure:

- Stage I evaluation of the patient's ability to tolerate an empty tray (placed in the mouth)

- Stage II evaluation of the patient's ability to tolerate a loaded tray (placed in the mouth)

- Stage III evaluation of the patient's ability to tolerate the impression in the mouth until the alginate set.

All results were recorded onto the patient's recording forms (with the help of their parents).

\section{Statistical analysis}

GSI and GPI scores were evaluated by the Wilcoxon signed rank test. Spearman rank correlation was applied to assess associations between GSI and GPI. The statistical significance level was determined at $\mathrm{p}<0.05$.

\section{RESULTS}

A total of 45 patients were unable to tolerate an upper alginate impression before the acupuncture. After the laser stimulation of acupuncture point CV 24 in Group A, 13 patients (86.6\%) were able to tolerate

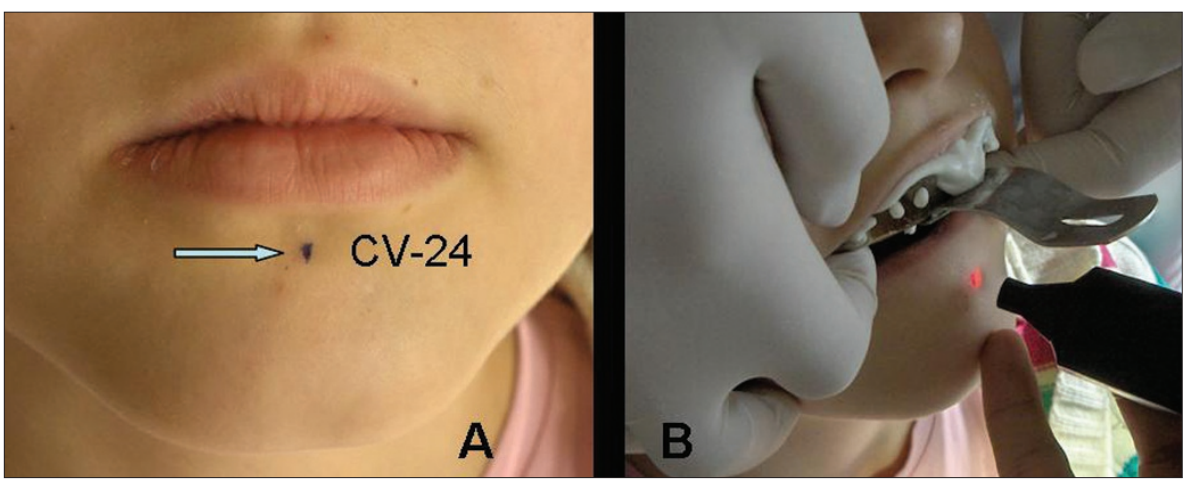

Fig. 1 The acupuncture point conception vessel 24 (CV 24). B. View of a red-light soft magnetic field laser stimulation on CV 24
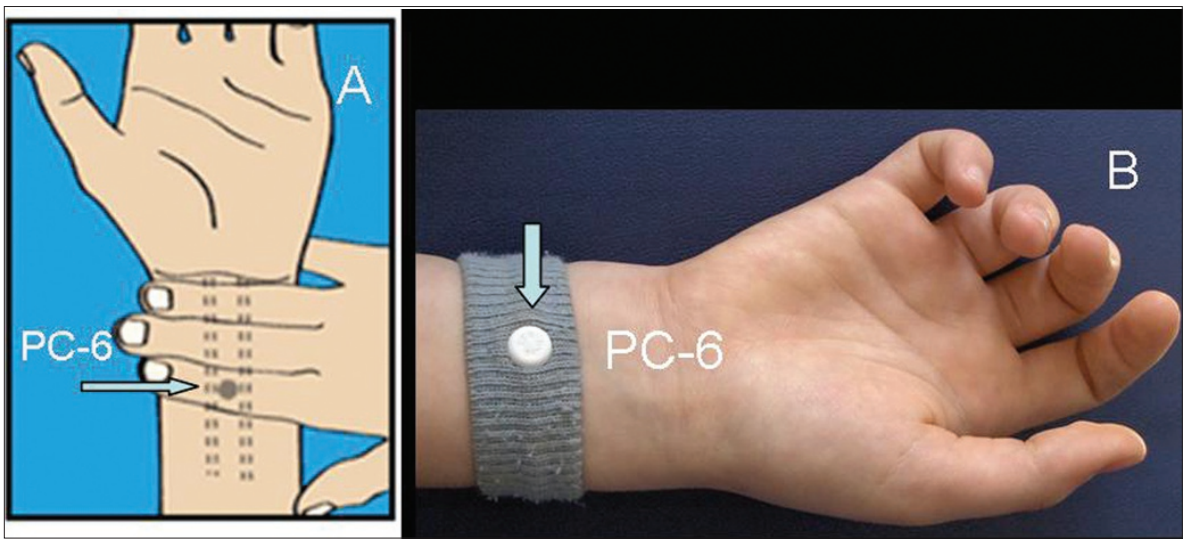

Fig. 2 The acupuncture point PC 6. B. The button on the band was placed over the PC 6

the impression taking whereas 2 patients (13.3\%) remained unable to tolerate the procedure. In Group B, however, 14 patients (93.3\%) could tolerate the impression. In Group C, none of the placebo patients could tolerate the impression taking.

GSI and GPI were used at the three stages of the impression taking procedure. The GSI and GPI scores are presented in Tables 1, 2 and 3. When one considers GSI and GPI scores at each stage, GPI scores were lower than GSI scores for both Group A and B. Furthermore, it was observed that this difference was statistically significant. ( $p<0.05)$. Note that in all three stages we have obtained $p<0.05$ significance in both Group A and Group B. However a significant decrease was observed in GPI scores relative to GSI scores, in Stage I in Group $C(p=0.006)$ (Table 3$)$. The differences between the GPI and GSI scores were not statistically significant at Stage II and III in Group C.

It was found that there was an increase in percentage improvement between the GSI and GPI scores before and after acupuncture in Group B when compared to those of Group A (Table 4). The average improvement between GSI and GPI scores

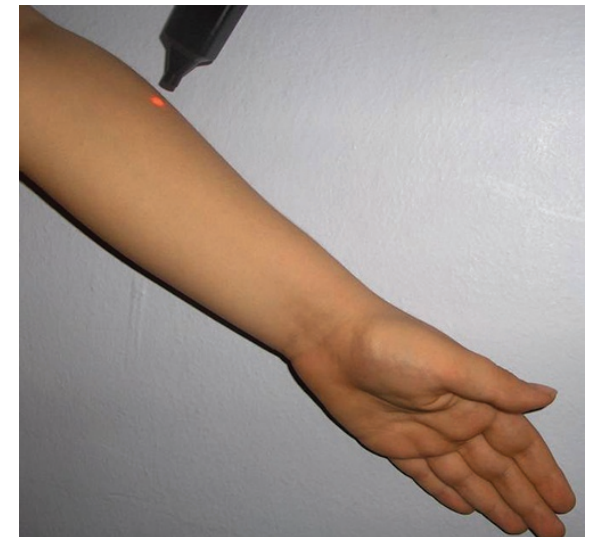

Fig. 3 The location of the placebo point

was 58.9\% in Group B while it was 37.9\% in Group A. The average improvement was $11.2 \%$ in Group C. No side effects were observed in patients receiving acupuncture

Orthodontists may have difficulty in taking upper alginate impressions in orthodontic patients with a gagging reflex. Various methods have been proposed to manage the gag reflex. ${ }^{3,8,17-18}$ Ansari ${ }^{16}$ recommended a secondary impression with injection type polyvinyl siloxane in a custom tray that will not stimulate the gag reflex, whereas

\section{DISCUSSION}


Friedman ${ }^{17}$ suggested that instructing the patients to extend their tongue may reduce the gag reflex by moving the point of stimulus to the tongue tip.

Acupressure and acupuncture are accepted as rapid, simple and very reliable methods of controlling the gag reflex with no morbidity. Ear acupuncture has been reported as very successful by Fiske and Dickenson. ${ }^{3}$ Rosted ${ }^{18}$ recommended acupuncture to point CV 24; arm points (PC 6) have been reported as effective by Lu et al., ${ }^{6}$ although not by Chate. ${ }^{19}$ Ren $^{20}$ suggested applying the pressure at the point of PC 6 with the thumb for 5 to 20 min before taking impressions. Other studies $^{21,22}$ have showed that acupressure on the P6 point using a wrist band is an alternative method in the prevention of nausea.

The use of laser acupuncture in children with needle phobia has been suggested in several articles recently., ${ }^{7,90,12}$ Schlager et al. ${ }^{13}$ investigated the effect of $670 \mathrm{~nm}$ low-level lasers on the acupuncture point PC 6 in children with vomiting after strabismus surgery. They found that laser stimulation of acupuncture point PC 6 reduced the incidence of vomiting. Dune et al. ${ }^{25}$ sought to determine acustimulation (AS) effects on postoperative nausea and vomiting in children. They undertook a meta-analysis to include acupressure, acupuncture, laser acupuncture, and electrical stimulation (ETS). This metaanalysis showed that acupressure and acupuncture are effective treatment modalities in reducing postoperative vomiting in children.

The effect of laser stimulation of the acupuncture point CV 24 and the combination of acupressure and acupuncture was investigated in this study. The PC 6 was chosen as an acupressure point. The reason for the usage of both CV 24and PC 6 in Group B was to investigate whether the two points have a synergistic effect.

Previous studies have showed that CV 24 and PC 6 were effective acupuncture points in patients with gagging reflex. Compared to needle acupuncture, acupressure and laser acupuncture allow less painful stimulation, hence acupressure and laser acupuncture were considered in this study. A low-level laser has also no known side effects. Our results showed that painless laser acupuncture stimulation of CV 24 point reduced the gag reflex without side effects. Rosted et al. ${ }^{15}$ used needle acupuncture on the acupuncture point CV 24 in patients with a gagging reflex. They found that CV 24 had a greater than $80 \%$ success rate in facilitating impression taking. The success rates in the study was 86.6\% for Group A, which was similar to the those of Rosted et al. ${ }^{15}$

Although a reduction in gagging reflex in both Groups A and B at the three stages was observed, there was a statistically significant decrease in GPI scores relative to GSI scores at Stage I for the placebo group (Group C). The tray was empty and the GSI scores were not remarkable $(3.5 \pm 0.6)$ at Stage I. The decrease in the GPI scores may depend on psychological factors in patients in Group C. Note that psychological and behavioral therapies are one of the methods used to cure the gag reflex.,4

The mode of action in controlling gag reflex through acupuncture is not fully understood. It covers the spectrum of the gag reflex from the mild end of nausea, to the severe end that culminates in vomiting. The gag reflex is assumed to be controlled from the vomiting centre. More recent studies indicate that multiple brain stem sites mediate the act of vomiting (or emesis) and that no isolated centre exists. ${ }^{26,27}$ The most likely cause of a somatically induced gag reflex during dental treatment is the activation of trigger zones in the posterior region of the oral cavity, which are innervated by the glossopharyngeal nerve (IX). On the other hand the anterior part of oral cavity is innervated by the trigeminal nerve $(\mathrm{V})$, which is not considered to take part in the gagging reflex. However there are close connections in the points between the cranial nerves V, IX, and X. ${ }^{28}$ After both stimulation of acupuncture point CV 24 and PC 6, the impulses ascend to centres in the mid-brain, in particular the nucleus of the raphe magnus ( $\mathrm{nRm})$. The nucleus of the raphe magnus is the main source of serotonin (5-HT) in the brain, ${ }^{28}$ which is metabolised to, among others, $\beta$-endorphine, which may have an anti-emetic function. It has been shown that acupuncture accelerates the synthesis of 5-HT, and it is likely that the serotonin mechanism takes part in the control of the gagging reflex. It could be concluded that both points CV 24 and PC 6 have a synergistic effect, because it was found that there was an increase in the percentage improvement between GSI and GPI in Group B compared to the results of Group A and C. However Dundee et al. ${ }^{29}$ found that the stimulation of PC 6 alone was insignificant in preventing postoperative vomiting in children after strabismus surgery. Barseum et al. ${ }^{30}$ showed that acupressure in adult patients reduced the nausea but not vomiting.

\section{CONCLUSION}

The laser stimulation of acupuncture point CV 24 was found to be an effective method in the treatment of orthodontic patients with gagging reflex. It was observed that there was an increase in the percentage improvement between the GSI and GPI utilising a combination of acupuncture and acupressure. Therefore it could be concluded that CV 24 and PC 6 have a synergistic effect.

1. Miles T S, Nauntofte B, Svenson P (eds). Clinical oral physiology. pp 245-254. Copenhagen: Quintessence Publishing Co Ltd, 2004.

2. Roth G I, Calmes R. Oral biology. pp 69. St Louis: Mosby; 1981.

3. Fiske J, Dickenson C. The role of acupuncture in controlling the gagging reflex using a review of ten cases. Br Dent J 2001: 190: 611-613.

4. Dickinson $C M$, Fiske J. A review of gagging problems in dentistry: 2 . Clinical assessment and management. Dent Update 2005; 32: 74-80.

5. Thayer M L. The use of acupuncture in dentistry. Dent Update 2007; 34: 244-250.

6. Lu D P, Lu G P, Reed J F 3rd. Acupuncture/acupressure to treat gagging dental patients: a clinical study of anti-gagging effects. Gen Dent 2000; 48: 446-452.

7. Rösler A, Otto B, Schreiber-Dietrich D, Steinmetz $H_{\text {, }}$ Kessler K R. Single-needle acupuncture alleviates gag reflex during transesophageal echocardiography: a blinded, randomised, controlled pilot trial. J Altern Complement Med 2003: 9: 847-849.

8. Litscher $\mathrm{G}$. Ten years evidence-based high-tech acupuncture - a short review of peripherally measured effects. Evid Based Complement Alternat Med 2009; 6: 153-158

9. Gottschling S, Meyer S, Gribova I et al. Laser acupuncture in children with headache: a double-blind, randomized, bicenter, placebo-controlled trial. Pain 2008; 137: 405-412.

10. Pothman $R$, Yeh $H \mathrm{~L}$. The effects of treatment with antibiotics, laser and acupuncture upon chronic maxillary sinusitis in children. Am J Chin Med 1982; 10: 55-58.

11. Zhou Y C. An advanced clinical trial with laser acupuncture anesthesia for minor operations in the oro-maxillofacial region. Lasers Surg Med 1984; 4: 297-303

12. Nedeljković M, Ljustina-Pribić R, Savić K. Innovative approach to laser acupuncture therapy of acute obstruction in asthmatic children. Med Preg/ 2008; 61: 123-130.

13. Schlager A, Offer T, Baldissera I. Laser stimulation of acupuncture point $\mathrm{P} 6$ reduces postoperative vomiting in children undergoing strabismus surgery. $\mathrm{Br} J$ Anaesth 1998: 81: 529-532.

14. Litscher G. Ten years evidence-based high-tech acupuncture part 3: a short review of animal experiments. Evid Based Complement Alternat Med advance online publication November 5 2008; doi:10.1093/ecam/nen034.

15. Rosted P, Bundgaard M, Fiske J, Pedersen A M. The use of acupuncture in controlling the gag reflex in 
patients requiring an upper alginate impression: an audit. Br Dent J 2006; 201: 721-725

16. Ansari I H. Management for maxillary removable partial denture patients who gag. J Prosthet Dent 1994; 72: 448.

17. Friedman M H, Weintraub M I. Temporary elimination of gag reflex for dental procedures. J Prosthet Dent 1995; 73: 319.

18. Rosted P. Use of acupuncture in dentistry. Aust Dent J 1998; 43: 437.

19. Chate R A. Acupuncture for controlling gag reflex? BrDent J 2001; 191: 537

20. Ren X. Making an impression of a maxillary edentulous patient with gag reflex by pressing caves. J Prosthet Dent 1997; 78: 533.

21. Lee M Y, Min H S. Effects of the Nei-Guan acupressure by wrist band on postoperative nausea and vomiting after middle ear surgery. Taehan Kanho Hakhoe Chi 2008; 38: 503-512.

22. Can Gürkan O, Arslan H. Effect of acupressure on nausea and vomiting during pregnancy. Complement Ther Clin Pract 2008; 14: 46-52.

23. Dune LS, Shiao S Y. Metaanalysis of acustimulation effects on postoperative nausea and vomiting in children. Explore (NY) 2006; 2: 314-320.

24. Hornby P J. Central neurocircuitry associated with emesis. Am J Med 2001; 111(Suppl 8A): 106-112. 25. Andrews $P$ L, Hawthorn J. The neurophysiology of vomiting. Baillieres Clin Gastroenterol 1988; 2: $141-168$.

26. Brodal P. The central nervous system. Structure and function. 3rd ed. USA: Oxford University Press, 2003.

27. Dundee J W, McMillan C M. P6 acupressure and postoperative vomiting. Br J Anaesth 1992; 68: 225-226.

28. Barsoum G, Perry E P, Fraser I A. Postoperative nausea is relieved by acupressure. J R Soc Med 1990; 83: 86-89. 